\title{
Bi-objective Optimization of a Single Machine Batch Scheduling Problem with Energy Cost Consideration
}

\author{
Shijin Wang ${ }^{\mathrm{a}}$, Ming Liu ${ }^{\mathrm{a}}$, Feng $\mathrm{Chu}^{\mathrm{b}}$, Chengbin $\mathrm{Chu}^{\mathrm{a}, \mathrm{c}}$ \\ ${ }^{a}$ Department of Management Science $\mathbb{B}$ Engineering, School of Economics 8 \\ Management, Tongji University, Shanghai 710049, P.R. China \\ ${ }^{b}$ Laboratory IBISC, University of Evry-Val dÉssonne, Evry 91020, France \\ ${ }^{c}$ Laboratoire Génie Industriel, Centrale Supélec, Université Paris-Saclay, Grande Voie \\ des Vignes, 92290 Châtenay-Malabry, France
}

\begin{abstract}
With the increasing energy price, the rapid growth of electricity demand and severe challenges for sustainable development, energy-efficient scheduling is becoming more and more important for power-intensive manufacturing industry, especially for batch production companies. This paper investigates a bi-objective single machine batch scheduling problem with non-identical job sizes, the time-of-use (TOU) electricity prices, and different energy consumption rates of the machine. The first objective is to minimize the makespan and the second is to minimize the total energy costs, by considering both the machine utilization and the economic cost. The problem is formulated as an integer programming model. Then, an exact $\epsilon$-constraint method is adapted to obtain the exact Pareto front. To deal with large scale problems, based on decomposition ideas, two heuristic methods are developed to obtain approximate Pareto fronts. Computational experiments on randomly generated instances show the effectiveness of the methods. A study case of a real-world glass manufacturing company is also conducted to show that the proposed methods are promising for practical usages.
\end{abstract}

Keywords:

Batch scheduling, Bi-objective optimization, Energy consumption cost, Makespan, Single machine

Corresponding author: mingliu@tongji.edu.cn (Ming Liu)

Preprint submitted to Journal of Cleaner Production

June 27, 2016

(C) 2016. This manuscript version is made available under the Elsevier user license http://www.elsevier.com/open-access/userlicense/1.0/ 


\section{Introduction}

The issue of batch scheduling optimization has attracted significant research attention, since on the one hand, many practical applications are highly related to batch-processing including chemical processes (Seid and Majozi, 2014; Fabre et al., 2011; Liu et al., 2010; Méndez et al., 2006), testing process in burn-in oven in the semiconductor manufacturing (Jula and Leachman, 2010; Sung et al., 2002; Sung and Choung, 2000), heat-treating ovens (Melouk et al., 2004). On the other hand, many batch scheduling problems are NP-hard (Potts and Kovalyov, 2000), even for most singlemachine environment, which makes them difficult to be solved in theoretical perspective.

However, in existing literature, few researches consider the energy cost for batch scheduling optimization even though most of batch processes are power-intensive industries and the electricity cost accounts for 10-50\% of the final product costs (Cheng et al., 2015). Meanwhile, energy cost is continuously rising (Kilian, 2008; Liu et al., 2015) due to the rapid and ongoing growth of energy demand. For example, demand for electric power in China grew 41\% from 2000 to 2007 (Fisher-Vanden et al., 2015). Moreover, energy consumption is one of the main reasons for the environment pollution due to the increasing amount of the green house gas emissions, in particular, the carbon dioxide $\left(\mathrm{CO}_{2}\right)$ (Ding et al., 2016). For example, about $28 \%$ greenhouse gas emissions in U.S.(Mouzon, 2008), about 18-20\% $\mathrm{CO}_{2}$ emission in Germany (Luo et al., 2013), and at least $26 \%$ of the total $\mathrm{CO}_{2}$ emission in China (Liu et al., 2014a), are from the manufacturing energy consumption. Therefore, it is highly important to improve energy usage efficiency in manufacturing (especially for energy-intensive industries) to save energy cost and to reduce greenhouse gas emissions (Gahm et al., 2016; Giret et al., 2015; Merkert, et al., 2015; He et al., 2015; Gong et al., 2016).

In this paper, motivated by scheduling challenges of the glass decoration and ceramization in the manufacturing, we study a single machine batch scheduling problem with non-identical job sizes. The key resource of the manufacturing process is a furnace (i.e., a batch machine), which is used to accommodate and ceramize glasses. The furnace has a fixed capacity (or space) that can accommodate different types of glasses with different sizes for simultaneous processing, i.e., glasses are processed in batches. The total size of the glasses in each batch should not exceed the furnace space capacity. Two objectives of the glass firm are considered: the makespan and the total 
energy cost. The reasons are as follows: (i) a minimum makespan usually implies a high utilization of the furnace since in the process the furnace is the bottleneck resource, and (ii) energy takes a large portion of the total costs in the glass firm.

The contributions of the paper can be summarized as follows:

(i) A bi-objective single machine batch scheduling problem is derived from a real-world glass manufacturing environment and an integer programming model is formulated by considering the decision makings of batch creation, batch sequence and furnace temperature choice;

(ii) An exact $\epsilon$-constraint method is adapted to obtain the exact Pareto front;

(iii) Two constructive heuristic methods are proposed based on the idea of two-stage hierarchical problem separations, to obtain good approximate Pareto fronts in a reasonable computation time;

(iv) Performance of the methods are validated by computational experiments and a case study from the real-world glass manufacturing.

The remainder of the paper is organized as follows. Section 2 reviews the relevant literature. Section 3 describes the problem and develops an integer program. Section 4 introduces the exact $\epsilon$-constraint method. Section 5 proposes two constructive heuristic methods based on the concept of twostage hierarchical batching and sequencing-temperature choice subproblem separations. The computational experiments and a study case are conducted to evaluate the proposed methods in Section 6. Finally, Section 7 concludes the paper and gives out the future research directions.

\section{Literature review}

In recent years, due to the energy shortage and the recognition of urgency in reducing energy consumption, there are more and more researches on energy-efficient scheduling in different manufacturing settings, including parallel machine (e.g., Li et al., 2016; Wang et al., 2015; Ji et al., 2013; Shabtay and Kaspi, 2006), job shop (e.g., Zhang and Chiong, 2016; Liu et al., 2015; Lei and Guo, 2015; Liu et al., 2014a), flow shop (e.g., Mansouri et al., 2016; Ding et al., 2016; Lin et al., 2015), and hybrid flow shop (e.g., Tang et al.,2015; Dai et al., 2013; Luo et al., 2013; Bruzzone et al., 2012). 
Readers can refer to recent survey papers by Gahm et al.(2015), Rager et al.(2015) and Giret et al.(2016) for more information. In this section, we mainly review the researches that are most highly related to this study, i.e., single machine scheduling with energy consideration, with and without batch setting.

Mouzon and Yildirim (2008) showed that large amounts of energy is wasted if machines can not be fully utilized and they investigated a bi-objective single machine scheduling problem to minimize the total energy consumption and the total tardiness. They developed a hierarchical bi-level framework in which a greedy randomized multi-objective adaptive search meta-heuristic to obtain an approximate Pareto front. Later, Yildirim and Mouzon (2012) studied a bi-objective single machine scheduling problem to minimize the energy consumption and the total completion time, in which the machine can be switched from idle to offline. They developed a multi-objective genetic algorithm to obtain an approximate Pareto front within reasonable computation time. Liu et al. (2014b) investigated a single machine scheduling problem with deterministic job arrival time to optimize two objectives: the total completion time and the total carbon dioxide emission. The Non-dominant Sorting Genetic Algorithm (NSGA-II) is applied to obtain an approximate Pareto front. Both Yildirim and Mouzon (2012) and Liu et al.(2014b) suggest that other conflicting objectives should be extensively investigated.

Shrouf et al. (2014) established a mathematical model for a single machine scheduling problem to minimize the total energy consumption costs. In the problem, the continuous changes in energy price, the energy consumption of each machine status, and the energy consumption of transitions between status are simultaneously considered. A genetic algorithm and an analytical solution are developed. Che et al. (2016) investigated a single machine scheduling under TOU policy to minimize the total electricity cost. They developed a new continuous-time mixed-integer linear programming (MILP) and proposed an efficient greedy insertion heuristic to solve the problem. However, in Shrouf et al.(2014) and Che et al.(2016), only energy-related objective is considered, performance indicator of production like makespan is not considered.

The main differences between these researches and this study are threefolds: (i) Different scheduling objectives with economic and environmental indicators are considered. We consider the makespan and the total energy cost simultaneously. (ii) Methods are significantly different. In these researches, only meta-heuristic methods are applied, while both exact and 
heuristic methods are proposed in this study. (iii) More importantly, batch scheduling is not considered in these researches, though batch productions are widely applied in industry and energy-intensive in many practical cases. Batch scheduling incurs extra decisions including how to form a batch and when to start a batch, which makes the problem more complicated but more practical.

Recently, there are some researches in single machine batch scheduling with energy cost consideration. Oron (2011) studied a single machine batch scheduling problem in which the job processing time is controlled by the resource (like energy) allocated on it. The relationship between the amount of resource allocation and job's processing time is a non-linear and convex function. Makespan and total completion time are considered as objectives, separately. However, only single objective is considered. Yue and You (2013) addressed a bi-objective batch scheduling problem with economic and environmental concerns. The two objectives are to maximize the productivity and to minimize the environmental impact per functional unit. They developed a bi-objective optimization model and proposed an $\epsilon$-constraint method to solve it. However, no heuristic or meta-heuristic methods are developed, which limits its application for practical large-scale problems. Cheng et al. (2015) studied a single machine batch scheduling problem to minimize two objectives: makespan and total electricity costs. A bi-objective model is proposed. An $\epsilon$-constraint method is applied to find the Pareto front and a fuzzy logic-based approach is used to get the preferable solution. However, in their study, each job has same size.

These researches provide significant insights on single machine energyefficient scheduling with batch. However, compared with abundant researches without batch consideration, this direction are still unexplored, whereas batch processes are power-intensive industries and the electricity cost accounts for $10-50 \%$ of the final product costs. Therefore, it is worth for further research.

\section{Problem statement and modeling}

\subsection{Production process}

The scheduling problem is originated from a special glass-ceramic man-

ufacturing shop floor. The schematic manufacturing process of this kind of glass is shown in Figure 1. Once production orders are placed by customers, raw glass already fabricated are cut into the customers' desired sizes. Then, 
the edges of each glass are smoothed and beveled. Next, small and large holes are drilled for future controlling knobs installation. After cleaning, customized logos and graphs are printed on the glass immediately followed by drying process. In the last step, each piece of glass is heated to a certain high degree, which makes the glass change at the molecular level and the glass is partially crystallizing, such that the special glass-ceramic is formed.

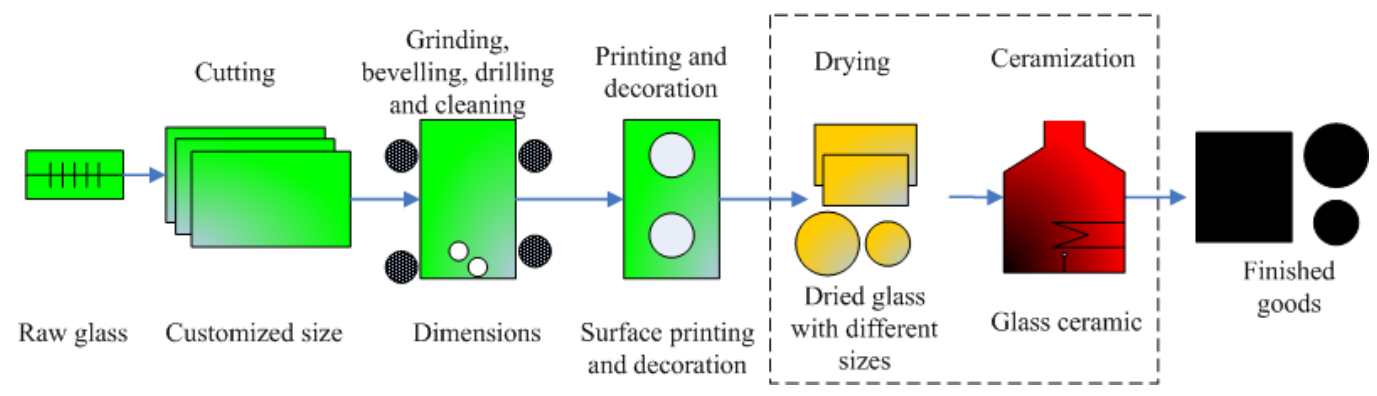

Figure 1: Schematic illustration of a special glass production process

In this study, we focus on the manufacturing process of the glass ceramization (highlighted by dashed line rectangular in Figure 1), in which a single furnace is used to process the glasses in batch. During the process of ceramization, pallets are used to hold different sizes of glasses. The question is how to form a batch and how to schedule each batch to minimize the makespan and the total energy cost.

\subsection{Problem description and formulation}

There is a set $K$ with $m$ different types of jobs (glass orders), each type of job has its size $s_{i}, i \in K=\{1,2, \ldots, m\}$. The total number of jobs is $N$, among which $n_{i}$ is the number of jobs with type $i$, where $i \in K$ and $\sum_{i \in K} n_{i}=N$. These jobs are to be processed on a batch heat-treating furnace within a scheduling horizon of $|T|$ periods (the duration of a period is a smallest interval on the time horizon), where $T$ denotes the set of all time periods. The number of batches $|B|$ is to be determined, where $B$ is the set of batches. The furnace has a size restriction with maximum size $S$. Only a batch with the total size not larger than $S$ can be processed by the furnace.

The temperature of the furnace can be adjusted between two levels: high and low. The energy consumption rates of the furnace are $E_{H}$ and $E_{L}$ for the high and low temperature, respectively. In general, $E_{H}>E_{L}$. If the furnace 
is not in processing, the energy consumption is zero. Once the temperature is set, it can not be changed until a batch is completed. Moreover, under the TOU policy, the unit energy price per period is variable and set as $c_{t}$, where $t \in T$. The processing time of a job of type $i$ for the high and low temperature is $p_{i}^{h}$ and $p_{i}^{l}, i \in K$, respectively. In general, $p_{i}^{h}$ and $p_{i}^{l}$ lasts for several periods and $p_{i}^{h}<p_{i}^{l}$. For the same job type, the processing time is the same. For a temperature level, the processing time of a batch is determined by the longest processing time of the jobs in that batch since it is acceptable for a glass ceramization to be kept in the furnace for a longer time than its specified heating time but not for a shorter time. It is assumed that the processing of a batch is non-preemptive and non-resumable, i.e., a batch processing can not be interrupted until it is finished and each of jobs in the batch must be entirely processed.

The scheduling decisions are to be made: (i) to form batches such that the furnace size is not violated, (ii) to determine the starting time of each batch in the scheduling period, and (iii) to determine the temperature level at the start of each batch, in order to minimize the makespan and the total energy costs simultaneously. Using the three-field notation (Graham et al., 1979), the problem can be denoted by $1, T O U|B|\left\{C_{\max }, T E C\right\}$, where ' $T O U$ ' represents the time-of-use policy of energy price, ' $B$ ' represents the batch processing, ' $C_{\max }$ ' represents the makespan, and ' $T E C$ ' represents the total energy cost.

In the following the mathematical model is formulated. First, the parameters are summarized and the decision variables are defined.

\section{Indices:}

$b, q$ : batch index, $b, q \in B$;

$i$ : job type index;

$j$ : job index of type $i, j=1, \ldots, n_{i}$, where $n_{i}$ is the number of jobs of type $i$

$t$ : time period index.

\section{Parameters:}

$K$ : a set representing $m$ different types of jobs;

$n_{i}$ : number of jobs of type $i, i \in K$; 
$m$ : number of different types of jobs, $m=|K|$;

$N$ : total number of jobs, i.e., $N=\sum_{i=1}^{m} n_{i}$;

$B$ : set of batches (i.e., $B=\{1, \ldots, N\}$, even though some batches may be empty), and $|B|$ denotes the number of batches;

$S$ : the fixed maximum size of the heat-treating furnace;

$s_{i}$ : size of job type $i, i \in K$;

$T$ : the set of time periods, and $|T|$ denotes the length of the planning horizon;

$E_{H}$ : amount of energy consumption during a period when the furnace is working in high temperature (i.e., the energy consumption rate of the furnace in high temperature);

$E_{L}$ : amount of energy consumption during a period when the furnace is working in low temperature;

$p_{i}^{h}$ : processing time of a job of type $i$ in the high temperature;

$p_{i}^{l}$ : processing time of a job of type $i$ in the low temperature. We assume that the $p_{i}^{l}$ is prolonged from $p_{i}^{h}$ proportionally with a ratio $g \geq 1$, i.e., $p_{i}^{l}=g \cdot p_{i}^{h}$;

$c_{t}$ : unit energy price for time period $t \in T$;

$M$ : a large enough positive integer.

\section{Decision variables:}

$C_{\max }$ : the makespan, i.e., the maximum completion time;

$x_{i j}^{b}$ : equal to 1 if job $j$ of type $i$ is assigned to batch $b, 0$ otherwise;

$y^{b}$ : equal to 1 if batch $b$ is formed with at least one job, 0 otherwise, i.e., no job is assigned to batch $b$;

$S T^{b}:$ start time of batch $b$ on the furnace;

$C T^{b}$ : completion time of batch $b$ on the furnace; 
$\beta^{b q}$ : equal to 1 if batch $b$ is processed before $q, 0$ otherwise;

$\alpha_{t}^{b}$ : equal to 1 if batch $b$ is processing at period $t, 0$ otherwise;

$\tau_{h}^{b}$ : equal to 1 if batch $b$ is processed with high temperature, 0 otherwise.

$\tau_{l}^{b}$ : equal to 1 if batch $b$ is processed with low temperature, 0 otherwise.

$P^{b}$ : processing time of batch $b$;

$z_{i j}^{b h}$ : equal to 1 if job $j$ of type $i$ is assigned to batch $b$ and that batch is with high temperature, 0 otherwise (That is, $z_{i j}^{b h}=1$ if and only if $x_{i j}^{b}=1$ and $\tau_{h}^{b}=1$.);

$z_{i j}^{b l}$ : equal to 1 if job $j$ of type $i$ is assigned to batch $b$ and that batch is with low temperature, 0 otherwise (That is, $z_{i j}^{b l}=1$ if and only if $x_{i j}^{b}=1$ and $\left.\tau_{l}^{b}=1.\right)$;

$\gamma_{t h}^{b}$ : equal to 1 if batch $b$ is processed at period $t$ with high temperature, 0 otherwise (That is, $\gamma_{t h}^{b}=1$ if and only if $\alpha_{t}^{b}=1$ and $\tau_{h}^{b}=1$ );

$\gamma_{t l}^{b}$ : equal to 1 if batch $b$ is processed at period $t$ with low temperature, 0 otherwise (That is, $\gamma_{t l}^{b}=1$ if and only if $\alpha_{t}^{b}=1$ and $\tau_{l}^{b}=1$ ).

The mathematical formulation for the problem is as follows:

$(\boldsymbol{I P})$

$$
\begin{array}{r}
\min f_{1}=\sum_{t \in T} c_{t}\left[\sum_{b \in B}\left(\gamma_{t h}^{b} E_{H}+\gamma_{t l}^{b} E_{L}\right)\right] \\
\min \quad f_{2}=C_{\max } \\
\text { s.t. } \quad \sum_{b \in B} x_{i j}^{b}=1, \quad \forall i \in K, j=1, \ldots, n_{i} \\
\sum_{i \in K} \sum_{j \in\left\{1,2, \ldots, n_{i}\right\}} s_{i} x_{i j}^{b} \leq y^{b} S, \quad \forall b \in B \\
\tau_{h}^{b}+\tau_{l}^{b}=1, \quad \forall b \in B
\end{array}
$$




$$
\begin{aligned}
& z_{i j}^{b h} \geq 1-\left(2-x_{i j}^{b}-\tau_{h}^{b}\right) M, \quad \forall i \in K, j=1, \ldots, n_{i}, b \in B \\
& z_{i j}^{b h} \leq \frac{x_{i j}^{b}+\tau_{h}^{b}}{2}, \quad \forall i \in K, j=1, \ldots, n_{i}, b \in B \\
& z_{i j}^{b l} \geq 1-\left(2-x_{i j}^{b}-\tau_{l}^{b}\right) M, \quad \forall i \in K, j=1, \ldots, n_{i}, b \in B \\
& z_{i j}^{b l} \leq \frac{x_{i j}^{b}+\tau_{l}^{b}}{2}, \quad \forall i \in K, j=1, \ldots, n_{i}, b \in B \\
& \sum_{t \in T} \alpha_{t}^{b}=P^{b}, \quad \forall b \in B \\
& P^{b} \geq z_{i j}^{b h} p_{i}^{h}+z_{i j}^{b l} p_{i}^{l}, \quad \forall i \in K, j=1, \ldots, n_{i}, b \in B \\
& t \geq S T^{b}-\left(1-\alpha_{t}^{b}\right)|T|, \quad \forall b \in B, t \in T \\
& t \leq C T^{b}-1+\left(1-\alpha_{t}^{b}\right)|T|, \quad \forall b \in B, t \in T \\
& S T^{b}+P^{b}=C T^{b}, \quad \forall b \in B \\
& C T^{b} \leq S T^{q}+\left(1-\beta^{b q}\right)|T|, \quad \forall b, q \in B, b \neq q \\
& \beta^{b q}+\beta^{q b}=1, \quad \forall b, q \in B, b \neq q \\
& C T^{b} \leq C_{\max }, \quad \forall b \in B \\
& \gamma_{t h}^{b} \geq 1-\left(2-\alpha_{t}^{b}-\tau_{h}^{b}\right) M, \quad \forall b \in B, t \in T \\
& \gamma_{t l}^{b} \geq 1-\left(2-\alpha_{t}^{b}-\tau_{l}^{b}\right) M, \quad \forall b \in B, t \in T
\end{aligned}
$$




$$
S T^{b}, C T^{b}, C_{\max } \in \mathbb{Z}_{+}, \quad \forall b \in B
$$

Expression (1) is to minimize the total energy consumption costs, which depends on the temperature level of the furnace and the energy price in every period. Expression (2) is to minimize the makespan $C_{\max }$, which is the maximum completion time of all batches. Constraint (3) ensures that a job $j$ of type $i$ is assigned to only one batch. Constraint (4) limits the total size of a batch not exceeding the maximum size (capacity) of the furnace. Constraint (5) ensures that a batch is processed either with the high temperature or the low one. Constraints (6) and (7) coordinate the values of $z_{i j}^{b h}, x_{i j}^{b}$ and $\tau_{h}^{b}$. Similarly, constraints (8) and (9) coordinate the values of $z_{i j}^{b l}, x_{i j}^{b}$ and $\tau_{l}^{b}$. Constraints (5)-(9) guarantee $z_{i j}^{b h}+z_{i j}^{b l}=1$. Constraint (10) guarantees that the processing time of a batch $b$ on the furnace is equal to its total working periods on the furnace. Constraint (11) determines the batch processing time. Constraints (12) and (13) make sure that the processing of batch $b$ on the furnace is not interrupted. Constraints (14) guarantees that the completion time of a batch is equal to the sum of its start time and its processing time. Constraint (15) imposes the precedence constraints of two consecutive batches on the furnace. Constraint (16) expresses the convexity on $\beta_{b q}$. Constraint (17) defines the range of $C_{\max }$. Constraints (18) and (19) are to linearize the terms $\gamma_{t h}^{b}=\alpha_{t}^{b} \tau_{h}^{b}$ and $\gamma_{t l}^{b}=\alpha_{t}^{b} \tau_{l}^{b}$, respectively. Constraints (20) and (21) give the ranges of the decision variables.

\subsection{Complexity of the problem}

Theorem 1. The problem $1, T O U|B|\left\{C_{\text {max }}, T E C\right\}$ is strongly NP-hard.

Proof. The special case of the problem $1|B|\left\{C_{\max }, T E C\right\}$ is the one with $c_{t}=1$ for every $t \in T$. In this case, the objective $T E C$ is equivalent to $C_{\max }$. Then, the problem is reduced to the single-machine batch scheduling problem with different job size requirements and makespan minimization, which has been proved to be strongly NP-hard (Uzsoy, 1994). Since this special case is strongly NP-hard, the result follows.

\section{An exact $\epsilon$-constraint method}

The $\epsilon$-constraint method is one of the most popular approaches for solving multiple objective optimization problems, especially for a bi-objective optimization problem like the problem $1, T O U|B|\left\{C_{\text {max }}, T E C\right\}$. In this section, 
we introduce an exact $\epsilon$-constraint method based on the concept of Pareto dominance.

\subsection{Pareto dominance and the $\epsilon$-constraint method}

For the minimization objectives, the Pareto dominance relationship denoted by $\prec_{P}$ can be defined as follows: a feasible solution $\mathbf{X}$ is said to dominate another feasible solution $\mathbf{Y}\left(\mathbf{X}, \mathbf{Y} \in \mathbf{Z}^{V}\right.$, which is a $V$-dimensional decision variable vector) denoted by $\mathbf{f}(\mathbf{X}) \prec_{P} \mathbf{f}(\mathbf{Y})$, if and only if, $f_{i}(\mathbf{X}) \leq f_{i}(\mathbf{Y})$ for $i=1,2, \ldots, p$ and $f_{i}(\mathbf{X})<f_{i}(\mathbf{Y})$ for at least one objective function of a given set of $p$ objective functions $\mathbf{f}(\cdot)=\left\{f_{1}, f_{2}, \ldots, f_{p}\right\}$. For the problem defined above, $p=2$.

With the concept of the Pareto dominance, all the non-dominated points in the objective space constitute the Pareto front, which provides a set of alternative solutions to decision makers for performance tradeoff.

The basic idea of the $\epsilon$-constraint method is to construct and solve a sequence of $\epsilon$-constraint problems by transforming one of the objectives as a constraint with a progressive reduction of $\epsilon$ value. To do this, the following points are needed (Cheng et al., 2015; Bérubé et al., 2009).

Ideal point: let $\mathbf{f}^{I}=\left(f_{1}^{I}, f_{2}^{I}\right)$ with $f_{1}^{I}=\min \left\{f_{1}(\mathbf{X})\right\}$ and $f_{2}^{I}=$ $\min \left\{f_{2}(\mathbf{X})\right\}, \mathbf{X} \in \mathbf{Z}^{\mathbf{V}}$;

Nadir point: let $\mathbf{f}^{N}=\left(f_{1}^{N}, f_{2}^{N}\right)$ with $f_{1}^{N}=\min \left\{f_{1}(\mathbf{X}): f_{2}(\mathbf{X})=f_{2}^{I}\right\}$ and $f_{2}^{N}=\min \left\{f_{2}(\mathbf{X}): f_{1}(\mathbf{X})=f_{1}^{I}\right\}, \mathbf{X} \in \mathbf{Z}^{\mathbf{V}}$;

Extreme point: $\mathbf{f}^{E}=\left\{\left(f_{1}^{I}, f_{2}^{N}\right),\left(f_{1}^{N}, f_{2}^{I}\right)\right\}$ are the set of two extreme points on the Pareto front.

Since both objectives of $T E C$ and $C_{\max }$ are integral for the problem under study, the following exact $\epsilon$-constraint method can be used to find the Pareto front (Cheng et al., 2015; Bérubé et al., 2009).

\subsection{An example solution with the exact $\epsilon$-constraint method}

To illustrate the problem, and also to demonstrate the conflicting relation between minimizing $T E C$ and $C_{\max }$, we solve a small problem with $m=3$, $|T|=30, E_{H}=5, E_{L}=2$ and $S=10$ by using the above exact $\epsilon$-constraint method in CPLEX 12.6. The detailed information of processing time $p_{i}^{h}$ and $p_{i}^{l}$ for high and low temperature, the number of jobs for each type $n_{i}$, the size 


\begin{tabular}{l}
\hline \hline Exact $\epsilon$-constraint method \\
1. Compute the ideal points $\mathbf{f}^{I}=\left(f_{1}^{I}, f_{2}^{I}\right)$ and Nadir points $\mathbf{f}^{N}=\left(f_{1}^{N}, f_{2}^{N}\right)$. \\
2. Set $\mathbf{F}^{\prime}=\left\{\left(f_{1}^{I}, f_{2}^{N}\right)\right\}$ and $\epsilon=f_{2}^{N}-\Delta(\Delta=1$ for this problem). \\
3. While $\epsilon \geq f_{2}^{I}$, do: \\
$\quad$ (a) solve the $\epsilon$-constraint problem with $C_{\max } \leq \epsilon$ as a constraint \\
and the $T E C$ as the single objective function to optimality, \\
and add the optimal solution value $\left(f_{1}^{*}, f_{2}^{*}\right)$ to $\mathbf{F}^{\prime}$. \\
(b) set $\epsilon=f_{2}^{*}-\Delta$. \\
4. Obtain the Pareto front $\mathbf{F}$ by removing dominated points from $\mathbf{F}^{\prime}$, if existing. \\
\hline \hline
\end{tabular}

Table 1: The detailed values for the example

\begin{tabular}{c|cccc}
\hline Job type $i$ & $p_{i}^{l}$ & $p_{i}^{h}$ & $n_{i}$ & $s_{i}$ \\
\hline 1 & 4 & 2 & 2 & 3 \\
2 & 4 & 2 & 2 & 5 \\
3 & 2 & 1 & 1 & 2 \\
\hline
\end{tabular}

Table 2: The energy prices at each period $c_{t}$

\begin{tabular}{cccccccccc}
\hline $\mathrm{t}$ & $c_{t}$ & $\mathrm{t}$ & $c_{t}$ & $\mathrm{t}$ & $c_{t}$ & $\mathrm{t}$ & $c_{t}$ & $\mathrm{t}$ & $c_{t}$ \\
\hline 1 & 5 & 7 & 1 & 13 & 3 & 19 & 5 & 25 & 1 \\
2 & 6 & 8 & 4 & 14 & 2 & 20 & 3 & 26 & 2 \\
3 & 3 & 9 & 2 & 15 & 6 & 21 & 1 & 27 & 5 \\
4 & 5 & 10 & 5 & 16 & 3 & 22 & 3 & 28 & 1 \\
5 & 3 & 11 & 5 & 17 & 4 & 23 & 4 & 29 & 5 \\
6 & 2 & 12 & 4 & 18 & 1 & 24 & 5 & 30 & 3 \\
\hline
\end{tabular}




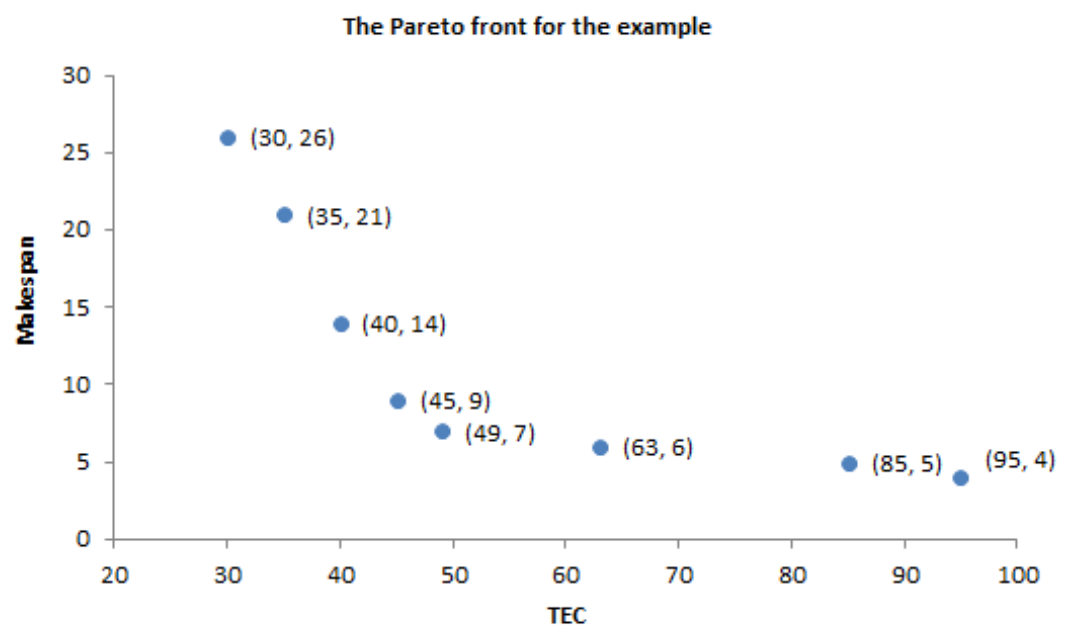

Figure 2: The Pareto front of the example problem

of each job type $s_{i}$ are given in Table 1 . The energy prices at each period $c_{t}$ are shown in Table 2.

The ideal point and nadir point are $\mathbf{f}^{I}=(30,4)$ and $\mathbf{f}^{N}=(95,26)$, respectively. Starting from one extreme point $(30,26)$, minimizing $T E C$ is considered as the objective and $C_{\max } \leq \epsilon$ as a constraint. Figure 2 represents the Pareto front, which includes 8 solutions. The results show that optimal $T E C$ and $C_{\max }$ cannot be optimized at the same time. On the other hand, it shows that if the schedule is only focused on the makespan, the energy consumption cost is much higher, even for this small scale problem.

\section{Constructive heuristic methods}

Due to the strongly NP-hardness of the problem, it's necessary to develop heuristics to find solutions in a reasonable computation time. Since the processing time of a batch is determined by the longest job included, it may be better to utilize the capacity of the machine fully. Therefore, an intuitive idea is: batch the jobs first and then sequence the batches. This helps to decompose the problem into two-stage hierarchical subproblems: the first one (denoted by upper subproblem) is how to batch the jobs and the second one (denoted by lower subproblem) is how to sequence the batches and determine the temperature for each batch. The whole structure of problem 
decomposition and the resulting two heuristic methods are shown in Figure 3 .

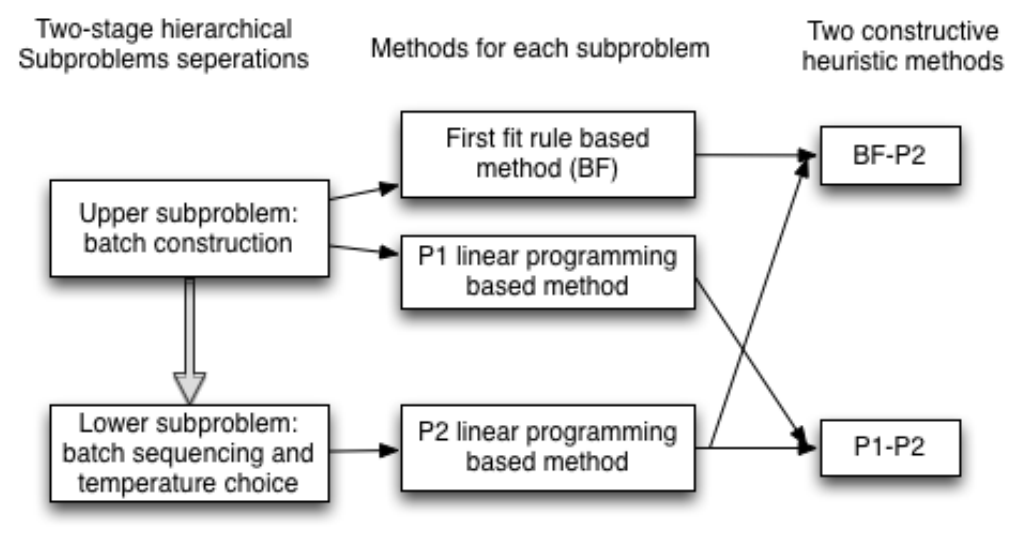

Figure 3: Structure of problem decomposition and two heuristic methods

\subsection{Methods for upper subproblem: batch construction}

We have two following batch construction methods. The first one is based on the batch first fit (FF) rule, and the second is based on optimization, i.e., solving an integer programming model by CPLEX.

\subsubsection{FF rule based batch construction}

For the first subproblem, the following method based on the batch first fit (FF) rule (Uzsoy, 1994) is designed. Here, let $S_{b}$ be the sum of the job sizes in batch $b$, and $G_{b}$ be the gap between the $S_{b}$ and $S$ (i.e., $G_{b}=S-S_{b}$ ). At the beginning, $S_{b}=0, G_{b}=S$ and $b=1$. Let $U$ be the set of jobs that are not assigned to any batch. At the beginning, $U$ includes all jobs.

The overall batching procedure (denoted by $B F$ hereafter) can be summarized as follows.

Step (1). first, all jobs are sequenced with a non-increasing order of $p_{i}^{h}, i=$ $1,2, \ldots, m$ (or in terms of $p_{i}^{l}$ since it is proportional to the $p_{i}^{h}$ with a fixed ratio). The set of sequenced jobs can be denoted by $U=$ $\{[1], \ldots,[k], \ldots,[N]\}$, where $k$ is the job sequenced on $k$ th position in $U$; 
Step (2). if $U=\emptyset$ terminate, otherwise, from the first unbatched job of the sequence set $U$, the first longest job $[k]$ with $s_{i}$ is selected. If $s_{i} \leq G_{b}$, go to Step (3); Otherwise, go to Step (4);

Step (3). put the job $[k]$ into batch $b, S_{b}=S_{b}+s_{i}, G_{b}=G_{b}-s_{i}, U=U-[k]$. Go to Step (2);

Step (4). if there is no job in $U$ whose size is less than or equal to $G_{b}, b=b+1$, $S_{b}=0, G_{b}=S$, go to Step (2); Otherwise, select a job with the longest processing time among jobs in $U$ whose sizes are not larger than $G_{b}$ (arbitrary breaking ties). Let job $[k]$ be the selected job and go to Step (3).

Table 3: The resulting batch information for the example

\begin{tabular}{cccc}
\hline Batch No. & Type $i$ in the batch & Job $j$ of type $i$ in the batch & Batch size \\
\hline 1 & 1 & 1 & 3 \\
1 & 1 & 2 & 6 \\
1 & 3 & 1 & 8 \\
\hline 2 & 2 & 1 & 5 \\
2 & 2 & 2 & 10 \\
\hline
\end{tabular}

Following the example in subsection 4.2, $S=10, s_{1}=3, s_{2}=5, s_{3}=2$, $n_{1}=n_{2}=2, n_{3}=1, N=5$. Using Step (1), $U=\{1,2,3,4,5\}$ corresponds to $J_{11}, J_{21}, J_{12}, J_{22}$ and $J_{13}$, respectively. Here, $J_{j i}$ represents job $j$ of type $i$. Then by following Step (2)-(4) of the BF procedure, the batches can be formed and they are shown in Table 3.

\subsubsection{An optimization based batch construction}

The second batch construction method is to generate batches to minimize the makespan, since makespan is one of the objectives. To achieve this, the following IP model can be formulated.

(IP for the upper subproblem (P1))

$$
\min \sum_{b \in B} P^{b}
$$

The constraints include: 
(3)-(4)

$$
\begin{gathered}
P^{b} \geq x_{i j}^{b} p_{i}^{h}, \quad \forall b \in B, i \in K, j=1, \ldots, n_{i} \\
x_{i j}^{b}, y^{b} \in\{0,1\}, \quad \forall b \in B, i \in K, j=1, \ldots, n_{i}
\end{gathered}
$$

In expression (23), the processing time of a batch is determined by the longest processing time of job with $s_{i}$ in batch $b$ with the high temperature, where $p_{i}^{h}$ is used to determine the batch processing time. The reason for doing this is that $p_{i}^{l}$ is assumed to be $g \cdot p_{i}^{h}, g>1$ in the problem. Therefore, in this model, the batch processing time for low temperature can be determined as $g \cdot P^{b}$ once $P^{b}$ is obtained through this model.

\subsection{An optimization based method for lower subproblem: batch sequence and temperature choice}

Once $|B|$ batches are formed, a batch can be regarded as an independent job with a batch processing time. Then, the lower subproblem is transformed into a single machine scheduling problem with TOU energy price and high/low temperature choice. In this subsection, we develop an integer programming optimization based method.

The lower subproblem can be formulated as follows.

\section{(IP for the lower subproblem (P2))}

The objectives:

$$
\text { (1) and (2) }
$$

The constraints include:

$$
\begin{gathered}
(5),(10),(12)-(19) \\
\sum_{b \in B} \alpha_{t}^{b} \leq 1, \quad \forall t \in T \\
P^{b}=\tau_{h}^{b} P^{H}+\tau_{l}^{b} P^{L}, \quad \forall b \in B \\
\alpha_{t}^{b}, \beta^{b q}, \tau_{h}^{b}, \tau_{l}^{b}, \gamma_{t h}^{b}, \gamma_{t l}^{b} \in\{0,1\}, \quad \forall b, q \in B, t \in T
\end{gathered}
$$

Inequality (25) ensures that in each period, only one batch can be processed on the machine. Since at some period, there may be no batch assigned, the sign $\leq$ is used. Constraint (26) is used to determine the processing time of a batch, in which $P^{L}=\max \left\{p_{i}^{l}\right\}$ and $P^{H}=\max \left\{p_{i}^{h}\right\}$, where $i$ means that job type $i$ in batch $b$.

Once the batch construction is done in upper subproblem, the $\epsilon$-constraint method can be used to find non-dominated solutions. 


\section{Computational experiments}

\subsection{Data generation}

The data required for an $1, T O U|B|\left\{C_{\max }, T E C\right\}$ problem consists of the machine energy consumption rate for high or low temperature $E_{H}, E_{L}$, the number of different job types $m$, the number of jobs for each type $n_{i}$, the processing time of a job type $i$ in the high temperature $p_{i}^{h}$, the proportional ratio rate $g$ for job processing time between low and high temperatures, the total number of scheduling periods $|T|$, the energy price for each time period $c_{t}$, the size of a job type $s_{i}$ and the total size of the machine space capacity $S$.

In the computational experiments, the parameters and their values are set as follows: the processing time of job type $i$ in the high temperature $p_{i}^{h}$ is taken from the uniform distribution between 1 and 5, which is denoted by $U[1,5]$. The proportional ratio rate $g$ for job processing time between low and high temperature is set as $g=2$. $c_{t}$ is taken from $U[1,6]$ and $s_{i}$ is taken from $U[1,5] . S=10, E_{H}=6, E_{L}=3$. The size of a problem is described by the combination of $\left\{m, n_{i},|T|\right\}$. For each combination, 30 problem instances are generated. For different sizes of problems, different values of $\left\{m, n_{i},|T|\right\}$ are taken. To ensure that there is a feasible solution, we take $|T| \geq \frac{m \times n_{i} \times \max \left\{p_{i}^{h}\right\} \times \max \left\{s_{i}\right\}}{S}$.

The exact $\epsilon$-constraint method for the IP model, the two optimisationbased methods (i.e., solve the integer programming for batch sequence and temperature choice), denoted by $B F-P 2$, and $P$ 1-P2, respectively are developed and implemented in $\mathrm{C}++$ and run on a $2.3 \mathrm{GHz}$ Intel Core i7 processor. The IP models are solved using CPLEX 12.6.

\subsection{Performance metrics}

It is necessary to use quality metrics to evaluate the performance of multiobjective algorithms. For approximate Pareto fronts, the following four metrics are used to compare the quality of two non-dominated fronts, $\mathbf{A}$ and $\mathbf{B}$, obtained by different algorithms. The first one is diversity (DVR) (c.f., Man-

souri et al., 2016), which represents the area covered by the two objective values, i.e., the product of the ranges of $C_{\max }$ and $T E C$.

$$
D V R_{\mathbf{A}}=\left(\max _{i \in \mathbf{A}} C_{\max }^{i}-\min _{i \in \mathbf{A}} C_{\max }^{i}\right) \times\left(\max _{i \in \mathbf{A}} T E C^{i}-\min _{i \in \mathbf{A}} T E C^{i}\right)
$$




$$
D V R^{\mathbf{A}}=\left|1-\frac{D V R_{\mathbf{A}}}{D V R_{\mathbf{S}^{*}}}\right|
$$

where $\mathbf{S}^{*}$ denotes a reference front (i.e., the Pareto-optimal front or a near Pareto-optimal front). If the Pareto front is not known, the two (or more if more than two methods used) fronts are combined and all the non-dominated solutions are selected to form the set $\mathbf{S}^{*}$. $\mathbf{A}$ is better than $\mathbf{B}$ if $D V R^{\mathbf{A}}<$ $D V R^{\mathbf{B}}$.

The second one is the Chebyshev distance metric (Bérubé et al., 2009), denoted by $C D$, which can be used to evaluate the distribution of front $\mathbf{A}$ and the distance of $\mathbf{A}$ to a reference front $\mathbf{S}^{*}$. The $C D$ measure of the front $\mathbf{A}$ is given by

$$
C D_{\mathbf{A}}=\frac{1}{\left|\mathbf{S}^{*}\right|} \sum_{z \in \mathbf{S}^{*}} \min _{z^{\prime} \in \mathbf{A}} \max \left(\frac{\left|z_{T E C}-z_{T E C}^{\prime}\right|}{z_{T E C}}, \frac{\left|z_{C_{\max }}-z_{C_{\max }}^{\prime}\right|}{z_{C_{\max }}}\right)
$$

$\mathbf{A}$ is regarded to be better than $\mathbf{B}$ if $C D_{\mathbf{A}}<C D_{\mathbf{B}}$

The third one is the number of solutions in front $\mathbf{A}$, denoted by $C R D$,

$$
C R D_{\mathbf{A}}=|\mathbf{A}|
$$

The computation time of different algorithms are also recorded for the fourth metric.

\subsection{Results for small scale problems}

Table 4 shows results of the exact $\epsilon$-constraint method for small scale problem instances with different combinations of $\left\{m, n_{i},|T|\right\}$. For each combination, 30 problem instances are generated by following the data generation scheme described in subsection 6.1. The columns in the table correspond to:

- $\left\{m, n_{i},|T|\right\}$ : the combination of $m, n_{i}$ and $|T|$;

- $N$ : the total number of jobs;

- Nsd: the number of problem instances solved optimally by the exact $\epsilon$-constraint method out of 30 instances within the time limit of 3600 seconds;

- $T M_{\mathbf{F}}^{\max }\left(T M_{\mathbf{F}}^{\min }\right)$ : the maximum (resp., minimum) total computation time (in seconds) for the problem instances solved optimally by the exact $\epsilon$-constraint method within the time limit of 3600 seconds; 
- $\operatorname{Avg}\left(\overline{T M}_{\mathbf{F}}\right)$ : the average computation time (in seconds) for the problem instances solved optimally by the exact $\epsilon$-constraint method within the time limit of 3600 seconds;

- $|\overline{\mathbf{F}}|$ : the average number of solutions in the exact Pareto front for the problem instances solved optimally within the time limit of 3600 seconds.

The reported results show that the exact $\epsilon$-constraint method can not address some problem instances within the given time limit even for those with small $m$ and $n_{i}$. Moreover, for the problem instances that can be solved within the time limit, the computation time of the exact $\epsilon$-constraint method varies largely even for the same $m$ and $n_{i}$, which can be shown by the $T M_{\mathbf{F}}^{\max }$ and $T M_{\mathrm{F}}^{\min }$ in the table. The results also show that generally with the total number of jobs $N$ and the length of the planning horizon $|T|$ increase, the number of the problem instances that can be solved to optimality within the time limits decreases, which can be demonstrated by the $N s d$ in the table.

Table 4: Statistics on the small scale problems for the exact $\epsilon$-constraint method

\begin{tabular}{ccccccc}
\hline$\left\{m, n_{i},|T|\right\}$ & $\mathrm{N}$ & $\mathrm{Nsd}$ & $T M_{\mathbf{F}}^{\max }$ & $T M_{\mathbf{F}}^{\min }$ & $\operatorname{Avg}\left(\overline{T M}_{\mathbf{F}}\right)$ & $|\overline{\mathbf{F}}|$ \\
\hline$\{2,1,10\}$ & 2 & 30 & 2275.39 & 0.089 & 203.65 & 2.67 \\
$\{2,1,30\}$ & 2 & 21 & 2835.35 & 0.46 & 959.85 & 4.05 \\
$\{3,1,15\}$ & 3 & 11 & 2911.57 & 0.76 & 976.35 & 2.30 \\
$\{3,1,30\}$ & 3 & 9 & 3438.74 & 238.23 & 2225.3 & 2.89 \\
$\{2,2,15\}$ & 4 & 8 & 3150.51 & 36.81 & 1242.05 & 3.50 \\
$\{2,2,30\}$ & 4 & 4 & 1376.11 & 0.76 & 633.97 & 4.25 \\
$\{2,2,50\}$ & 4 & 5 & 2751.80 & 39.32 & 2470.92 & 3.00 \\
$\{3,2,30\}$ & 6 & 2 & 2662.97 & 232.71 & 1655.22 & 3.50 \\
$\{3,2,50\}$ & 6 & 2 & 1092.71 & 941.97 & 1023.92 & 8.0 \\
\hline
\end{tabular}

On the other hand, the two heuristic methods: BF-P2 and P1-P2, spend much less computation effort for the problem instances (see Table 5), and all methods get the same Pareto front as the exact $\epsilon$-constraint method does for the problem instances that are solved optimally within the time limit. In the table, $\overline{T M}_{B F-P 2}$ and $\overline{T M}_{P 1-P 2}$ represent the average of the total computation time (in seconds) with the BF-P2 and the P1-P2 method, respectively. 
Table 5: Average total computation time for small scale problems with two heuristic methods

\begin{tabular}{cccc}
\hline$\left\{m, n_{i},|T|\right\}$ & $\mathrm{N}$ & $\overline{T M}_{B F-P 2}$ & $\overline{T M}_{P 1-P 2}$ \\
\hline$\{2,1,10\}$ & 2 & 0.03 & 0.04 \\
$\{2,1,30\}$ & 2 & 0.12 & 0.13 \\
$\{3,1,15\}$ & 3 & 0.06 & 0.07 \\
$\{3,1,30\}$ & 3 & 0.18 & 0.20 \\
$\{2,2,15\}$ & 4 & 0.10 & 0.18 \\
$\{2,2,30\}$ & 4 & 0.22 & 0.25 \\
$\{2,2,50\}$ & 4 & 0.22 & 0.25 \\
$\{3,2,30\}$ & 6 & 0.14 & 0.16 \\
$\{3,2,50\}$ & 6 & 0.41 & 0.43 \\
\hline
\end{tabular}

\subsection{Results for large scale problems}

The computational results of large scale problem instances are shown in Table 6-7. Table 6 summarizes the mean and standard deviation of the computation time of the two heuristic methods for the same problem instances. The $\epsilon$-constraint method can not get any solution with time limits of 3600 CPU seconds. The computation time of BF-P2 and P1-P2 are much less than those of the $\epsilon$-constraint method. Moreover, it can be observed that the computation time is affected by both number of total jobs, $N$, and the number of total planning horizon $|T|$. For the combination $\left\{m, n_{i},|T|\right\}=\{5,3,80\}$, $\left\{m, n_{i},|T|\right\}=\{5,3,100\}$ and $\left\{m, n_{i},|T|=\{6,3,100\}\right.$, BF-P2 and P1-P2 can only solve 26, 21 and 17 optimally out of 30 problem instances within the time limit of $3600 \mathrm{CPU}$ seconds. It should be noted that, in Table 6-7, the mean and standard deviation results are based on the problem instances that are solved optimally within the time limit.

Table 7 reports the mean and standard deviation of the DVR, CD and CRD of the two heuristic methods for the same problem instances. In terms of DVR, BF-P2 and P1-P2 are quite close. The P1-P2 method is relatively better than the BF-P2 method, since the BF-P2 has a larger diversity. For the $\mathrm{CD}$ value, we observe that $\mathrm{P} 1-\mathrm{P} 2$ is better. This may be because that $\mathrm{P} 1$ and P2 obtain the optimal solutions at both stages. In terms of CRD, the results suggest that CRD increases as the parameters of $N$ and $|T|$ increase. This can be explained by that more different batch sequences and time slots could be tested in the search space. BF-P2 and P1-P2 can obtain very close number of solutions. The BF-P2 is slightly better than the P1-P2 in terms 
of CRD.

Table 6: Mean (standard deviation) computation time on the large scale problems for the two heuristic methods

\begin{tabular}{cccc}
\hline$\left\{m, n_{i},|T|\right\}$ & $N$ & BF-P2 & P1-P2 \\
\hline$\{5,2,60\}$ & 10 & $30.57(93.48)$ & $29.32(84.53)$ \\
$\{5,2,80\}$ & 10 & $25.91(44.29)$ & $23.22(34.37)$ \\
$\{5,3,80\}$ & 15 & $423.15(676.21)$ & $279.01(489.32)$ \\
$\{5,3,100\}$ & 15 & $364.7(560.83)$ & $520.78(829.65)$ \\
$\{6,3,100\}$ & 18 & $880.26(730.2)$ & $971.38(853.84)$ \\
\hline
\end{tabular}

Table 7: Mean (standard deviation) of DVR, CD and CRD on the large scale problems for the two heuristic methods

\begin{tabular}{cc|cc|cc|cc}
\hline$\left\{m, n_{i},|T|\right\}$ & $N$ & $D V R^{B F-P 2}$ & $D V R^{P 1-P 2}$ & $C D_{B F-P 2}$ & $C D_{P 1-P 2}$ & $C R D_{B F-P 2}$ & $C R D_{P 1-P 2}$ \\
\hline$\{5,2,60\}$ & 10 & $0.004(0.02)$ & $\mathbf{0}(0)$ & $0.016(0.067)$ & $\mathbf{0}(0)$ & $9.37(3.92)$ & $9.37(3.94)$ \\
$\{5,2,80\}$ & 10 & $0.074(0.29)$ & $\mathbf{0 . 0 0 2}(0.008)$ & $0.021(0.06)$ & $\mathbf{0 . 0 0 2}(0.012)$ & $10.5(2.33)$ & $10.37(2.57)$ \\
$\{5,3,80\}$ & 15 & $0.035(0.115)$ & $\mathbf{0 . 0 0 9}(0.046)$ & $0.020(0.05)$ & $\mathbf{0}(0)$ & $12.96(4.03)$ & $12.5(3.71)$ \\
$\{5,3,100\}$ & 15 & $\mathbf{0 . 0 0 1}(0.004)$ & $0.003(0.016)$ & $0.003(0.015)$ & $\mathbf{0 . 0 0 1}(0.007)$ & $13.62(3.11)$ & $13.43(3.29)$ \\
$\{6,3,100\}$ & 18 & $0.008(0.031)$ & $\mathbf{0}(0)$ & $0.010(0.028)$ & $\mathbf{0}(0)$ & $13.88(3.92)$ & $13.77(4.02)$ \\
\hline
\end{tabular}

\subsection{A case study of glass production company}

The following case is originated from a real-world scheduling problem in a glass ceramization company located in Shanghai, China. Its data shares the common characteristics of the glass ceramization company. However, we have preprocessed the data for some commercial confidentiality reasons.

In the coming $|T|=60$ time periods (one time unit corresponds to 10 minutes), there are four types of glasses which should be processed on the glass tempering furnace. All these four kinds of glasses have same height $8 \mathrm{~mm}$. The detailed job information are described in Table 8, in which, type 1 and type 2 jobs are circle glasses, and type 3 and type 4 jobs are rectangular ones. In practice, the different glasses will be put on the specific pallet which will be rolled into the furnace for heating (see Figure 4). The furnace can process two pallets at a same time with each pallet comprising the same combination of different glasses. The schematic diagram of these job types on one pallet is shown in Figure 5. The power of the glass tempering furnace is $900 \mathrm{~kW}$ when it is running at $600^{\circ} \mathrm{C}$, and $660 \mathrm{~kW}$ when it is running at 
$450^{\circ} \mathrm{C}$. The capacity of the furnace is simplified as $S=24$. The main energy considered here is electricity and the electricity price for industry during summer periods is illustrated as follows (unit: RMB Yuan $/ \mathrm{kWh}$ ), due to the peak, normal and valley times.

$$
c_{t}= \begin{cases}0.3, & 0 \leq t<10 \\ 0.7, & 10 \leq t<22 \\ 1.2, & 22 \leq t<32 \\ 0.3, & 32 \leq t<44 \\ 0.7, & 44 \leq t<60\end{cases}
$$

Table 8: The detailed data for the studied case

\begin{tabular}{c|cccc}
\hline Job type $i$ & $p_{i}^{l}$ & $p_{i}^{h}$ & $n_{i}$ & $s_{i}$ \\
\hline 1 & 2 & 1 & 15 & 3 \\
2 & 4 & 2 & 13 & 6 \\
3 & 10 & 5 & 6 & 18 \\
4 & 8 & 4 & 6 & 12 \\
\hline
\end{tabular}

The computational results are shown in Figure 6. For the exact $\epsilon$ constraint method, no one solution can be obtained after 31823.64 CPU seconds. The approximate Pareto fronts of the BF-P2 and P1-P2 are the same, which includes 11 non-dominated solutions. It takes the BF-P2 and the P1-P2 methods 5648.05 and 6359.25 CPU seconds, respectively. The solutions along the front range from energy costs 4470 (RMB Yuan) to 3720 (RMB Yuan), and makespan 48 to 58. From the Pareto solutions $(4470,48)$ to $(3720,58)$, there is $16.8 \%$ energy cost reduction at the cost of $20.8 \%$ increase of the makespan. We also observe that, along the Pareto front, the marginal energy cost saving is 75 RMB Yuan (i.e., the cost is reduced by 75 RMB Yuan with the increase of one unit time of 10 minutes), which can provide production managers for trade-off analysis between the total energy costs and the makespan. The reason for the linear changes of the approximate Pareto fronts may be because that the electricity price is stepwise changed, not continuously changed. There is no non-dominated solution with a makespan larger than 58, which means that no energy cost savings can be further achieved 


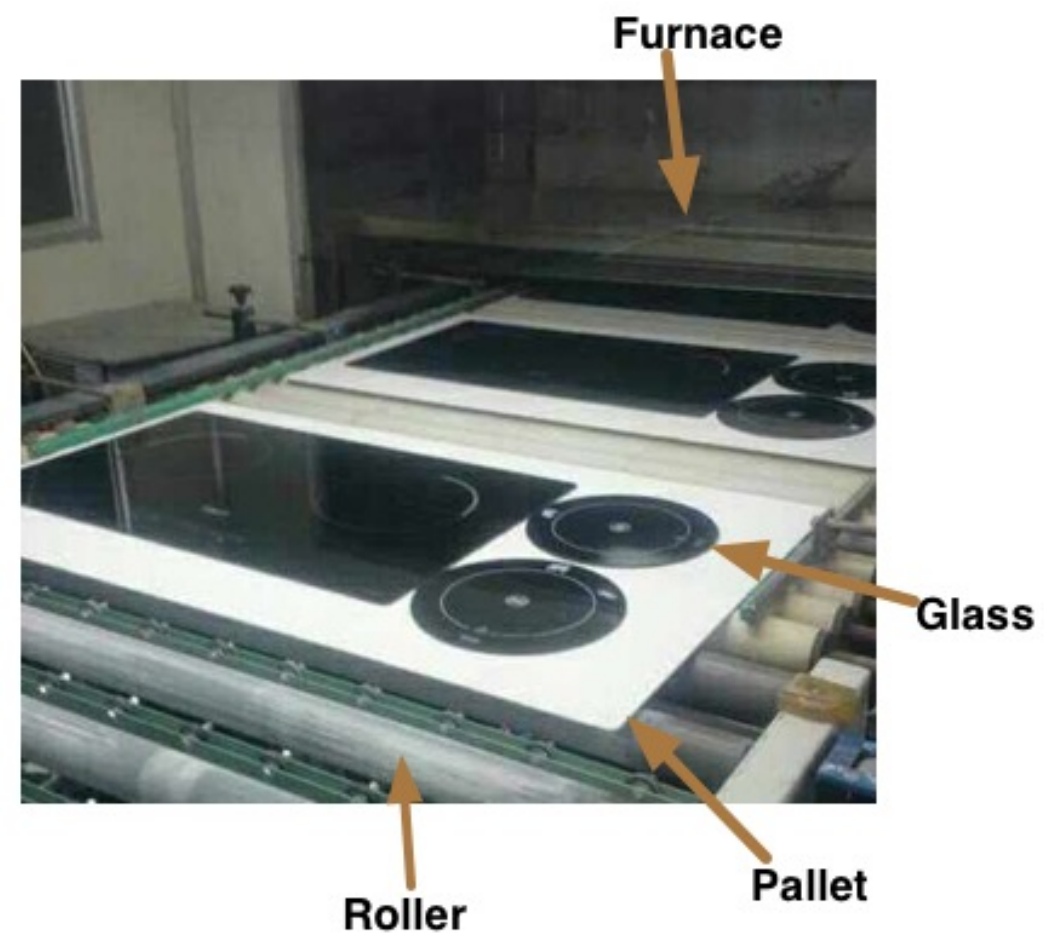

Figure 4: Two batches of glasses before rolling into the furnace 


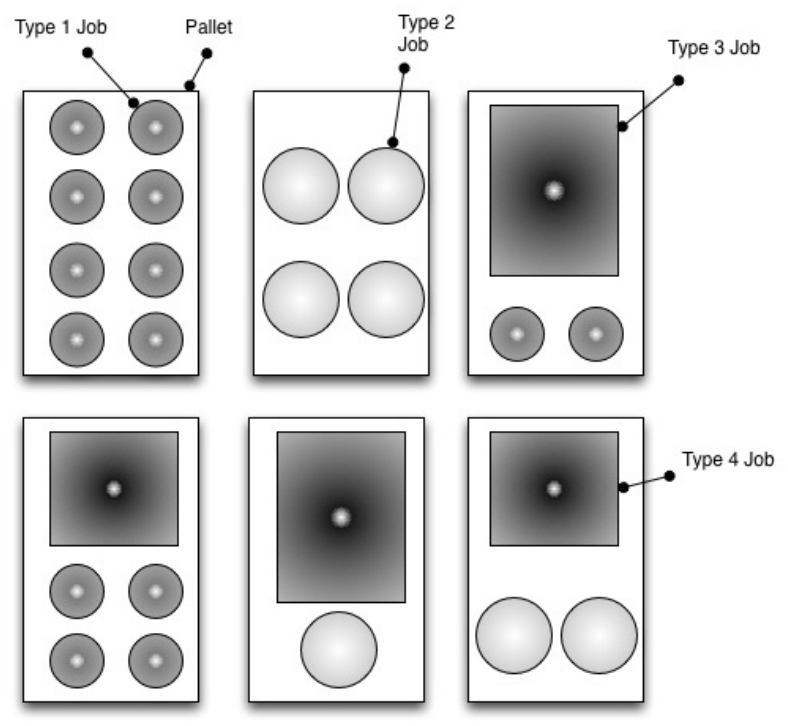

Figure 5: The examples of batch combinations of these four job types

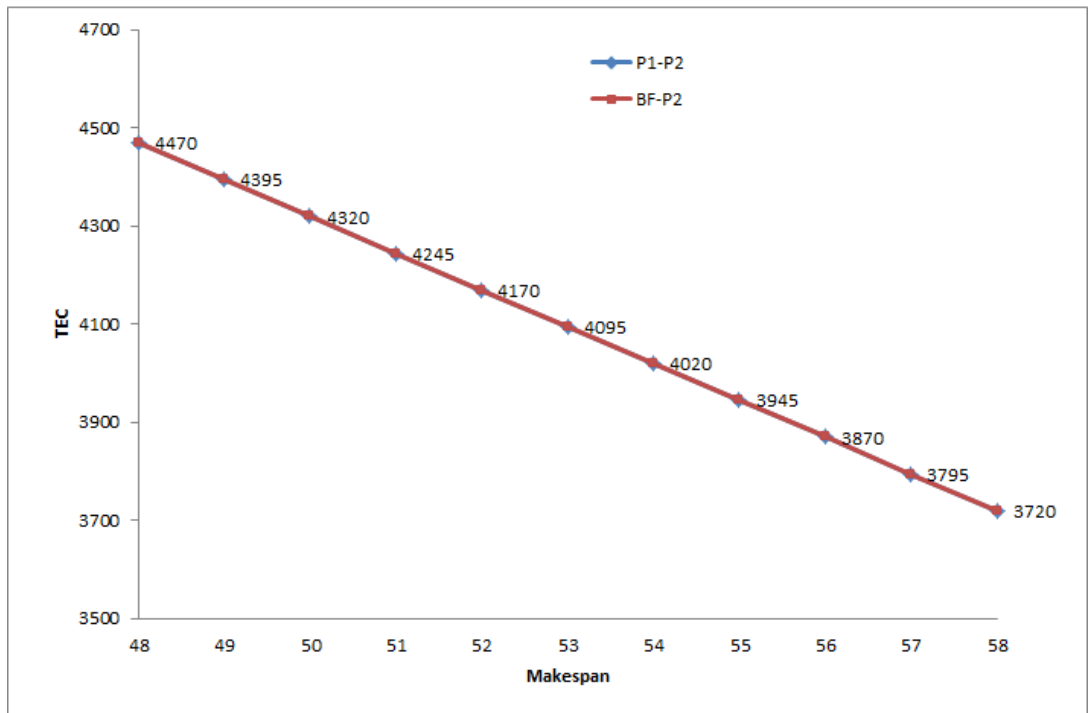

Figure 6: The approximate Pareto fronts obtained by the two heuristic methods for the studied case 
with the consumption of makespan. In addition, there is no solution with makespan less than 48. This is because that the optimal solution for batch formulation P1 is 48 .

The detailed batch formulation for the solution with the objective function values $(4470,48)$ is shown in Table 9 , where $P^{b}$ represents the batch processing time. It shows that there are 13 batches. In the schedule, the high temperature is selected for each batch. As for the start time, the first batch starts from time zero, and then it follows batch 2 , and so on until batch 13 , which leads to the solution with the objective values $(4470,48)$. For the solution with the objective function values $(3720,58)$, the batch formulation is the same as in Table 9, and also the high temperature is chosen for al1 batches. However, the start time $S T^{b}$ and completion time $C T^{b}$ of each batch are different, and they are shown in Table.10.

Table 9: The detailed batch information with minimum makespan 48

\begin{tabular}{c|ccc}
\hline Batch No. & Job type in the batch & Batch size & $P^{b}$ \\
\hline 1 & $\{2,3\}$ & 24 & 5 \\
2 & $\{2,3\}$ & 24 & 5 \\
3 & $\{2,3\}$ & 24 & 5 \\
4 & $\{2,3\}$ & 24 & 5 \\
5 & $\{2,3\}$ & 24 & 5 \\
6 & $\{2,3\}$ & 24 & 5 \\
7 & $\{4,4\}$ & 24 & 4 \\
8 & $\{4,4\}$ & 24 & 4 \\
9 & $\{4,4\}$ & 24 & 4 \\
10 & $\{2,2,2,2\}$ & 24 & 2 \\
11 & $\{2,2,2,1,1\}$ & 24 & 2 \\
12 & $\{1,1,1,1,1,1,1,1\}$ & 24 & 1 \\
13 & $\{1,1,1,1,1\}$ & 15 & 1 \\
\hline
\end{tabular}

\section{Conclusions}

This paper investigates a single machine batch scheduling problem to minimize two objectives simultaneously: the makespan and the total energy cost. The time-of-use energy price and temperature choice for batches are considered. A comprehensive mathematical formulation is presented. An $\epsilon$-constraint method is applied to get the exact Pareto front, and two 
Table 10: The detailed start time and completion time of each batch for the solution with objective vaules $(3720,58)$

\begin{tabular}{c|cc||c|cc}
\hline Batch No. & $S T^{b}$ & $C T^{b}$ & Batch No. & $S T^{b}$ & $C T^{b}$ \\
\hline 1 & 5 & 10 & 8 & 47 & 51 \\
2 & 51 & 56 & 9 & 39 & 43 \\
3 & 10 & 15 & 10 & 15 & 17 \\
4 & 17 & 22 & 11 & 56 & 58 \\
5 & 0 & 5 & 12 & 38 & 39 \\
6 & 32 & 37 & 13 & 37 & 38 \\
7 & 43 & 47 & & & \\
\hline
\end{tabular}

decomposition-based heuristic methods are used to get approximate Pareto fronts.

Computational experiments are conducted on problem instances randomly generated and a case study to evaluate the efficiency and effectiveness of the methods. The results show that for small scale problems, the heuristics can obtain exact Pareto front in much shorter time compared with the $\epsilon$ constraint method. For large scale problems and the practical size case, the P1-P2 and BF-P2 can get better solutions in terms of performance metrics with relatively short computation time. Therefore, as for managerial implications, we suggest the P1-P2 or BF-P2 method for production managers to solve middle and large scale problems, which help to find the trade-off between the furnace utilization (related to production efficiency) and the total energy cost (related to economic return).

For the future work, various multi-objective algorithms could be developed for this problem. The extension to multiple-machine environments like parallel machine is also interesting and practical, since for some glass manufacturing companies, there are more than one glass tempering furnace with similar heating functions.

\section{Acknowledgement}

The authors would like to thank the anonymous referees for their constructive comments. This work was supported by the National Science Foundation of China (NSFC) under Grants 71171149, 71428002, 71571134 and 71571135. This work was partially supported by the Cai Yuanpei Program 
between the French Ministries of Foreign and European Affairs and the Higher Education and Research and the Chinese Ministry of Education, under Grant (27927VE). The work was also supported by the Fundamental Research Funds for the Central Universities.

\section{References}

[1] Bérubé J.F., Gendreau M., Potvin J.Y. (2009) An exact $\epsilon$-constraint method for bi-objective combinatorial optimization problems: application to the traveling salesman problem with profits. European Journal of Operational Research, 194: 39-50.

[2] Brucker P., Kovalyov M.Y. (1996) Single machine batch scheduling to minimize the weighted number of late jobs. Mathematical Methods of Operations Research, 43: 1-8.

[3] Bruzzone A.A.G., Anghinolfi D., Paolucci M., Tonelli F. (2012) Energyaware scheduling for improving manufacturing process sustainability: a mathematical model for flexible flow shops. CIRP Annals - Manufacturing Technology, 61: 459-462.

[4] Che A., Zeng Y., Lyu K (2016) An efficient greedy insertion heuristic for energy conscious single machine scheduling problem under time-of-use electricity tariffs. Journal of Cleaner Production, 129: 565-577.

[5] Cheng J.H., Chu F., Chu C.B., Xia W.L. (2015) Bi-objective optimization of single-machine batch scheduling under time-of-use electricity prices. RAIRO Operations Research, in press.

[6] Dai M., Tang D.B., Giret A., Salido M.A., Li W.D. (2013) Energyefficient schedulingfora flexible flow shop using an improved geneticsimulated annealing algorithm. Robotics and Computer-Integrated Manufacturing, 29: 418-429.

[7] Ding J.Y., Song S.J., Wu C. (2016) Carbon-efficient scheduling of flow shops by multi-objective optimization. European Journal of Operational Research, 48(3): 758-771.

[8] Fabre F., Htreux G., Lann J.M.L., Zaraté P. (2011) Dynamic hybrid simulation of batch processes driven by a scheduling module. Computers and Chemical Engineering, 35: 2098-2112. 
[9] Fisher-Vanden, K., Mansur E.T., Wang Q. (2015) Electricity shortages and firm productivity: evidence from China's industrial firms. Journal of Development Economics, 114: 172-188.

[10] Gahm C., Denz F., Dirr M., Tuma A. (2016) Energy-efficient scheduling in manufacturing companies: a review and research framework. European Journal of Operational Research, 248(3): 744-757.

[11] Giret A., Trentesaux D., Prabhu V. (2015) Sustainability in manufacturing operations scheduling: a state of the art review. Journal of Manufacturing Systems, 37(1): 126-140.

[12] Gong X., Pessemier T.D., Joseph W., Martens L. (2016) A generic method for energy-efficient and energy-cost-effective production at the unit process level. Journal of Clearner Production, 113: 508-522.

[13] Graham R.L., Lawler E.L., Lenstra J.K., Rinnooykan A.H.G. (1979) Optimization and approxiamtion in deterministic sequencing and scheduling: a survey. Annals of Discrete Mathematics, 5: 287-326.

[14] He Y., Li Y.F., Wu T., Sutherland J.W.(2015) An energy-responsive optimization method for machine tool selection and operation sequence in flexible machining job shops, Journal of Cleaner Production, 87: 245254.

[15] Ji M., Wang J.Y., Lee W.C. (2013) Minimizing resource consumption on uniform parallel machines with a bound on makespan. Computers \& Operations Research, 40: 2970-2974.

[16] Jula P., Leachman R.C. (2010) Coordinated multistage scheduling of parallel batch-processing machines under multiresource constraints. Operations Research, 58(4): 933-947.

[17] Kilian, L. (2008) The economic effects of energy price shocks. Journal of Economic Literature 46, 871-909.

[18] Lei D.M., Guo X.P. (2015) An effective neighborhood search for scheduling in dual-resource constrained interval job shop with environmental objective. International Journal of Production Economics, 159: 296-303. 
[19] Li K.,Zhang X., Leung J.Y.T., Yang S.L. (2016) Parallel machine scheduling problems in green manufacturing industry. Journal of Manufacturing Systems, 38, 98-106.

[20] Lin W.W., Yu D.Y., Zhang C.Y., Liu X., Zhang S.Q., Tian Y.H., Liu S.Q., Xie Z.P. (2015) A multi-objective teaching-learning-based optimization algorithm to scheduling in turning processes for minimizing makespan and carbon footprint. Journal of Cleaner Production, 101: 337-347.

[21] Liu B., Wang L., Liu Y., Qian B., Jin Y.H. (2010) An effective hybrid particle swarm optimization for batch scheduling of polypropylene processes. Computers and Chemical Engineering, 34: 518-528.

[22] Liu C.G., Yang J., Lian J., Li W.J., Evans S., Yin Y. (2014b) Sustainable performance oriented operational decision-making of single machine systems with deterministic product arrival time. Journal of Cleaner Production, 85: 318-330.

[23] Liu Y., Dong H.B., Lohse N., Petrovic S., Gindy N. (2014a) An investigation into minimising total energy consumption and total weighted tardiness in job shops. Journal of Cleaner Production, 65: 87-96.

[24] Liu Y., Dong H.B., Lohse N., Petrovic S. (2015) Reducing environmental impact of production during a Rolling Blackout policy-a multi-objective scheduling optimisation approach. Journal of Cleaner Production, 102: 418-427.

[25] Luo H., Du B., Huang G.Q., Chen H.P., Li X.L. (2013) Hybrid flow shop scheduling considering machine electricity consumption cost. International Journal of Production Economics, 146: 423-439.

[26] Mansouri S.A., Aktas E., Besikci U. (2016) Green scheduling of a twomachine flowshop: trade-off between makespan and energy consumption. European Journal of Operational Research, 248(3): 772-788.

[27] Melouk S., Damodaran P., Chang P.Y. (2004) Minimizing makespan for single machine batch processing with non-identical job sizes using simulated annealing. International Journal of Production Economics, 87: $141-147$. 
[28] Méndez C.A., Cerdá J., Grossmann I.E., Harjunkoski I., Fahl M. (2006) State-of-the-art review of optimization methods for short-term scheduling of batch processes. Computers and Chemical Engineering, 30: 913946.

[29] Merkert L., Harjunkoski I., Isaksson A., Saynevirta S., Saarela A., Sand G. (2015) Scheduling and energy-industrial challenges and opportunities. Computers and Chemical Engineering, 72: 183-198.

[30] Mouzon, G. (2008) Operational Methods and Models for Minimisation of Energy Consumption in a Manufacturing Environment. Wichita State University.

[31] Mouzon G., Yildirim M.B. (2008) A framework to minimize total energy consumption and total tardiness on a single machine. International Journal of Sustainable Engineering, 1(2): 105-116.

[32] Oron D. (2011) Scheduling a batching machine with convex resource consumption functions. Information Processing Letters, 111: 962-967.

[33] Potts C.N., Kovalyov M.Y. (2000) Scheduling with batching: a review. European Journal of Operational Research, 120: 228-249.

[34] Raqer M., Gahm C., Denz F. (2015) Energy-oriented scheduling based on evolutionary algorithms. Computers \& Operations Research, 54: 218231.

[35] Seid E.R., Majozi T. (2014) Heat integration in multipurpose batch plants using a robust scheduling framework. Energy,71: 302-320.

[36] Shabtay D.,Kaspi M. (2006) Parallel machine scheduling with a convex resource consumption function. European Journal of Operational Research, 173: 92-107.

[37] Shrouf F., Ordieres-Mere J., Garcia-Sanchez A., Ortega-Mier M. (2014) Optimizing the production scheduling of a single machine to minimize total energy consumption costs. Journal of Cleaner Production, 67: 197207.

[38] Sung C.S., Choung Y.I. (2000) Minimizing makespan on a single burn-in oven in semiconductor manufacturing. European Journal of Operational Research, 120: 559-574. 
[39] Sung C.S., Choung Y.I., Hong J.M., Kim, Y.H. (2002) Minimizing makespan on a single burn-in oven with job families and dynamic job arrivals. Computers \& Operations Research, 29: 995-1007.

[40] Tang D.B., Dai M., Salido M.A., Giret A. (2015) Energy-efficient dynamic scheduling for a flexible flow shop using an improved particle swarm optimization. Computers in Industry, in press.

[41] Uzsoy R. (1994) Scheduling a single batch processing machine with nonidentical job sizes. International Journal of Production Research, 32(7): 1615-1635.

[42] Wang Y.C., Wang M.J., Lin S.C. (2015) Selection of cutting conditions for power constrained parallel machine scheduling. Robotics and Computer-Integrated Manufacturing, in press.

[43] Yildirim M.B., Mouzon G. (2012) Single-machine sustainable production planning to minimizing total energy consumption and total completion time using a multiple objective genetic algorithm. IEEE Transactions on Engineering Management, 59(4): 585-597.

[44] Yue D.J, You F.Q. (2013) Sustainable scheduling of batch processes under economic and environmental criteria with MINLP models and algorithms. Computers and Chemical Engineering, 54: 44-59.

[45] Zhang R., Chiong R. (2016) Solving the energy-efficient job shop scheduling problem: a multi-objective genetic algorithm with enhanced local search for minimizing the total weighted tardiness and total energy consumption. Journal of Clearner Production, 112(4): 3361-3375. 